RLM the presence of a resection margin $<1 \mathrm{~mm}$ was the only independent predictor of survival (OR 2.86; $\mathrm{p}<0.001)$.

Conclusion Overall 5-year survival following resection of both CLM and RLM is similar. Those factors which predict long-term survival following liver resection however differ and this may have implications for selecting those for intensive follow-up or who may be candidates for adjuvant therapy.

Competing interests None declared.

\section{PWE-144 ENDOSCOPIC ULTRASOUND IN THE EVALUATION OF LIVER HILAR PATHOLOGY}

doi:10.1136/gutjnl-2012-302514d.144

${ }^{1} \mathrm{~S}$ Putta, ${ }^{*} \mathrm{D}$ Croagh, ${ }^{3} \mathrm{R}$ Boulton, ${ }^{2} \mathrm{~S}$ Brahmall, ${ }^{4} \mathrm{C}$ Forde, ${ }^{4} \mathrm{~B}$ Mahon. ${ }^{1}$ Liver Medicine, Queen Elizabeth Hospital, Birmingham, UK; ${ }^{2}$ Liver surgery, Queen Elizabeth Hospital, Birmingham, UK; ${ }^{3}$ Department of Gastroenterology, Queen Elizabeth Hospital, Birmingham, UK; ${ }^{4}$ Department of Radiology, Queen Elizabeth Hospital, Birmingham, UK

Introduction Liver hilar pathology has traditionally been challenging to investigate as histological diagnosis is often difficult to obtain. The diagnostic yield of existing techniques including ERCP is suboptimal. Patients have consequently been managed in the absence of histological diagnosis, with the attendant hazards, including potential metal stent placement or unwarranted surgery in the absence of malignant disease. Upto $15 \%$ of patients with suspected biliary malignancy who undergo surgery are found to have benign disease. The utility of endoscopic ultrasound has not been established and our study aims to evaluate the role of EUS in this setting.

Methods This is a retrospective review of all patients with a hepatic hilar stricture and or mass, who were reviewed at the hepatobiliary multidisciplinary meeting between July 2006 and September 2011 and went on to have an EUS examination. Patients with presumed benign disease were followed until they underwent definitive surgery, or for 1-year with serial cross sectional imaging.

Results 95 patients with hilar lesions underwent 114 EUS examinations. $67(70 \%)$ patients were diagnosed to have malignant disease. 58 patients had biliary tarct cancer. EUS-FNA yielded a positive cytological diagnosis in $52(78 \%)$ patients. In 15 patients EUS cytology was false negative. 28 patients were diagnosed with benign disease. Factors that predicted malignant disease at EUS examination included the presence of a bile duct associated mass lesion ( $p$ value 0.0001) and an EUS morphological diagnosis of cancer ( $p$ value 0.03 ). The presence of "pathological" lymph nodes was not statistically significant ( $p$ value 0.79 ). Sensitivity in obtaining a cytological diagnosis, accuracy in defining benign and malignant disease, specificity and negative predictive value of EUS were $78 \%, 85 \%, 100 \%$, and $66 \%$ respectively. Metal stent insertion was contemplated in atleast two patients following cross sectional imaging but was abandoned after EUS confirmed the benign nature of their condition.

Conclusion In the largest series to date we report high sensitivity $(80 \%)$ for EUS cytology in the diagnosis of malignant disease and accuracy (85\%) in distinguishing malignant from benign disease. EUS had a significant impact on the clinical management of our patients, including prevention of potential metal stent placement in atleast two patients who were eventually diagnosed with benign disease.

\section{Competing interests None declared.}

\section{REFERENCE}

1. Fritscher-Ravens A, Broering DC, Knoefel WT, et al. EUS-guided FNA of suspected hilar cholangiocarcinoma in potentially operable patients with negative brush cytology. Am J Gastroenterol 2004;99:45-51.

\section{PWE-145 PROGNOSTIC FACTORS INFLUENCING SURVIVAL AFTER LIVER RESECTION FOR COLORECTAL METASTASIS}

doi:10.1136/gutjnl-2012-302514d.145

S K P John, * A Vallance, S Rehman, S Robinson, R Charnley, B Jaques, D Manas S White. Hepatobiliary Surgery, Newcastle Hospitals NHS Foundation Trust, Newcastle upon Tyne, UK

Introduction A variety of factors have been identified in the literature which influence survival following resection of colorectal liver metastases (CRLM). The aim of this study was to identify those factors which influence survival in patients undergoing resection of CRLM in a UK centre.

Methods All patients having liver resection for CRLM during an 11-year period up to 2011 were identified from a prospectively maintained database and relevant clinical data retrieved from case records. Prognostic factors analysed included tumour size $(>5 \mathrm{~cm}$ or $<5 \mathrm{~cm}$ ), lymph node status of primary tumour, margin positivity $\mathrm{R} 1$ $(<1 \mathrm{~mm}$ ) or R0, neo-adjuvant chemotherapy (for liver), tumour differentiation, number of liver metastasis (4 or more), preoperative CEA (>200 or $<200)$ and whether metastases were synchronous (ie, diagnosed $<12$ months) or metachronous to the primary tumour. Overall survival (OS) was compared with Kaplan-Meier plots, log rank test. Multi-variate analysis was performed using Cox regression model (SPSS V.19). $\mathrm{p}<0.05$ considered significant.

Results 432 patients underwent resection of CRLM during this period (67\% male; mean age 64.5 years). The overall 5 -year survival in this series was $43 \%$. A pre-op CEA $>200$ was present in $10 \%$ of patients and was associated with a poorer 5 -year OS $(24 \%$ vs $45 \%$; $\mathrm{p}<0.001)$. A resection margin $<1 \mathrm{~mm}$ was present in $16 \%$ of patients and this had a negative impact on 5 yr OS $(15 \%$ vs $47 \%$; $\mathrm{p}<0.001)$. Tumour differentiation, number, size, presence of biliary or vascular invasion, relationship to primary disease, nodal status of primary, or the use of neoadjuvant chemotherapy had no impact on OS. Multi-variate analysis identified only the presence of a positive resection margin $(O R$ 1.75; $\mathrm{p}<0.05$ ) and a pre-op $C E A>200$ (OR 1.88; $\mathrm{p}<0.01$ ) as independent predictors of poorer $\mathrm{OS}$

Conclusion Despite the wide variety of prognostic factors reported in the literature we were only able to identify a pre-op CEA $>200$ and the presence of tumour within $1 \mathrm{~mm}$ of the resection margin as being of value in predicting survival. These variables are likely to identify patients who may benefit from intensive follow-up to enable early adjuvant chemotherapy postoperatively.

Competing interests None declared.

\section{PWE-146 BILIARY INVASION IN COLORECTAL LIVER METASTASIS-DOES IT MATTER?}

doi:10.1136/gutjnl-2012-302514d.146

S K P John,* S Rehman, B Harrison, S Robinson, B Haugk, D Manas, S White Hepatobiliary Surgery, Newcastle Hospitals NHS Foundation Trust, Newcastle upon Tyne, UK

Introduction Vascular invasion of colorectal liver metastasis following liver resection has a detrimental effect on patient outcome. However there are few studies specifically looking at the significance of biliary invasion in colorectal liver metastasis (CRLM). The aim of this study was therefore to evaluate the presence of biliary invasion and patient outcomes in those having liver resection for CRLM.

Methods All patients having liver resection for colorectal liver metastasis during an 11-year period up to 2011 were identified from a prospectively maintained database. The unit follows standardised pathological reporting for CRLM where biliary and vascular invasion are assessed and documented. Missing information was 